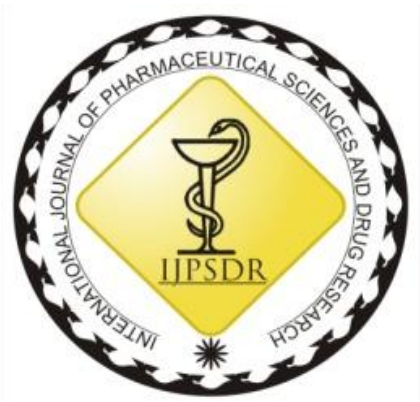

ISSN: 0975-248X

RESEARCH ARTICLE CODEN (USA): IJPSPP

$(\mathrm{cc})$ EY-NG-SA

\title{
To Study the Significance of Processing Variables Using Quality by Design for Optimization of Nanoparticulate System of Cilnidipine
}

\author{
Arti Bagada1, K. R. Vadalia ${ }^{2}$, M. K. Raval ${ }^{1 *}$, Dolly Gadhia1 \\ ${ }^{1}$ Department of Pharmaceutical Sciences, Saurashtra University, Rajkot, Gujarat, India \\ ${ }^{2}$ Atmiya Institute of Pharmacy, Rajkot, Gujarat, India
}

Copyright (C) 2019 Arti Bagada et al. This is an open access article distributed under the terms of the Creative Commons AttributionNonCommercial-ShareAlike 4.0 International License which allows others to remix, tweak, and build upon the work non-commercially, as long as the author is credited and the new creations are licensed under the identical terms.

\section{ABSTRACT}

This investigation aimed to prepare Cilnidipine Nanoparticles by nanoprecipitation ultrasonication method and to study the significance of processing variables by applying quality by design. Cilnidipine is fourth-generation dual $\mathrm{L} / \mathrm{N}$-type $\mathrm{Ca}^{2+}$ channel blocker used for the management of hypertension. It is BCS class-II drug exhibiting lower aqueous solubility, which tends to lower bioavailability. The combination of Poloxamer 188 and Tween 80 was used as a stabilizer. The design of the experiment is one of the tools of Quality by design. Plackett-Burman design was applied for the screening of processing variables, which are significant for the method. The processing variables screened were stirring speed, antisolvent ratio, drug concentration, polymer concentration, stabilizer concentration. The effect of each parameter evaluated by particle size, entrapment efficiency, and drug release at 10 minutes of prepared Nanoparticles of Cilnidipine. Analysis of variance and Pareto-plot of Plackett-Burman design were utilized to find the significance of the factor and extent of the effect. The surface morphology of Cilnidipine Nanoparticles was studied by SEM. The Pareto plot, as well as statistical analysis of design, had shown that the Concentration of drug, solvent: antisolvent ratio and concentration of poloxamer 188 were the significant parameters for the method. The stabilizer concentration, the stirring speed, and the antisolvent ratio had a negative effect of while the concentration of drug has a positive effect on the particle size of Nanoparticles and drug release at 10 minutes and positive effect of entrapment efficiency of Cilnidipine Nanoparticles. The Cilnidipine Nanoparticles were characterized by FTIR and DSC analysis.

Keywords: Processing variables, Nanoparticulate System, Cilnidipine, Plackett-Burman Design.

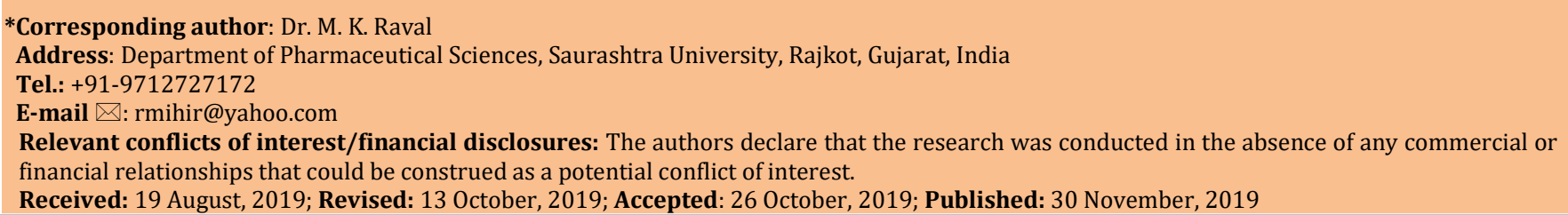

\section{INTRODUCTION}

Cilnidipine (CLN) is a new dihydropyridine Cachannel blocker that had given excellent effect clinically on cardiovascular diseases. [1] Cilnidipine is diester 1,4dihydropyridine-3,5-dicarboxylic acid and it blocks both L and N-type ca- channels. [2] It only possesses reno, neuro and cardioprotective action out of all antihypertensive drugs. It is a yellow, odorless crystalline powder having molecular weight 492 $\mathrm{g} / \mathrm{mol}$. CLN is included in BCS class 2 drug which has 
low aqueous solubility and high permeability. The drug with poor aqueous solubility shows the poor dissolution rate which resulted in low oral bioavailability from an effective dose of $10 \mathrm{mg}$. ${ }^{[3]}$ Development of poorly water-soluble drugs formulations is always a challenging task for formulators and In the future also $40 \%$ of newly developed drugs expected to be poorly soluble. ${ }^{[4]}$

The solubility can be enhanced by different traditional strategies like micronization, co-solvency technique, utilizing permeation enhancers, utilizing surfactant but they may have limited use. even though considerable results have been obtained by formulating hydrophobic drugs utilizing techniques like emulsions, liposomes, microemulsions, solid dispersion technology and inclusion complexes with cyclodextrins, but for all drugs, anyone approaches not applicable. At present, the need is for a unique technology that may overcome the problems related to the formulation of hydrophobic drugs which improve their clinical efficacy and optimize their therapy related to pharmacoeconomics. [4]

The drug powder is converted to drug nanoparticles by basic two techniques like bottom-up technology and top-down technology. [5] A pharmaceutical nanosuspension is characterized as all-around finely scattered strong medication particles in a fluid vehicle for either oral furthermore, topical and Parenterals use. The molecule estimate circulation of the strong particles in nanosuspension is typically not exactly less than one micron. Nanosuspension has demonstrated its capability to beat the issues related to the less watersoluble drugs and lipid dissolvable medications with the effortlessness and the points of interest over different strategies. ${ }^{[6]}$

The present study aimed to increase the dissolution rate of less water-soluble drugs CLN by the nanoparticulate system. Nanoparticles were prepared by the nanoprecipitation method. This technique was developed and patented by Fessi and co-workers. ${ }^{[7-8]}$ In these techniques the Nano size is achieved by one single step. This method requires one solvent in which drug is highly soluble and one solvent in which drug is insoluble. Important thing is that these two solvents must be miscible with each other. By the means of rapid desolvation of the polymer particles nanoprecipitation observed. At the point when the polymer-containing dissolvable polymer diffuses into the dispersed medium, it precipitates out following entrapment of drug. The moment arrangement of the Nanoparticles is because of the Marangoni impact, which is a result of interfacial turbulences occur at the interface of the dissolvable and the non-dissolvable solvent and result from complex and cumulated marvels, for example, stream, dispersion and surface strain varieties. ${ }^{[9]}$ Nano size range of 100-300 nm with narrow unimodal distribution can be achieved by this technique. Entrapment efficiency value up to $100 \%$ can be obtained due to zero drug leakage towards the outer medium. [7, 10]

In this method, many processing variables were required to be optimized. To select the critical processing variables which have a significant effect on Cilnidipine Nanoparticles the Plackett Burman design (PB Design) was utilized. The objective of this design is the screening of important parameters among the various parameters. The PB Design is a two-level factorial design and the impact of every factor was given by the equation

$$
\text { Eai }=2(\Sigma \text { Hai }-\Sigma \text { Lai }) / \mathrm{N}-\text { - - }^{-} \text {(I) }
$$

Where Eai is the concentration effect of a particular variable, Hai is the response at the higher level, Lai is the response at the lower level and $\mathrm{N}$ is the total number of trials.

A positive sign in the model for a reaction demonstrates an impact that favors and the negative sign shows a reverse connection between reactions. In the Plackett-Burman experimental design, the linear equation is expressed as

$\mathrm{R}=\mathrm{B}_{0}+\mathrm{B}_{1} \mathrm{~A}_{1}+\mathrm{B}_{2} \mathrm{~A}_{2}+\mathrm{B}_{3} \mathrm{~A}_{3}+\mathrm{B}_{4} \mathrm{~A}_{4}+\ldots+\mathrm{B}_{\mathrm{N}} \mathrm{A}_{\mathrm{N}} \ldots$. . (II) Where $R$ is the response, $B 0$ is the constant and $B 1$, $\mathrm{B} 2 \ldots \mathrm{BN}$ are the coefficients of the variables $\mathrm{A} 1, \mathrm{~A} 2, \ldots$ AN. [11-15]

\section{MATERIALS AND METHODS \\ Materials}

Cilnidipine was obtained as a gift sample from Laksh Finchem, Anand. The poloxamer 188 was obtained as a gift sample from BASF, Germany. The tween 80 was purchased from Loba Chemical Pvt. Ltd., India.

\section{Preparation of Cilnidipine Nanoparticles}

The formulation of nanosuspension was performed by the nanoprecipitation ultrasonication method. Precipitation with sonication was used to prepare nanosuspension. The specific amount of stabilizers Poloxamer 188 and tween 80 were dissolved into distilled water. The CLN drug was dissolved in dimethyl sulphoxide (DMSO) to prepare an organic solution of the drug. This solution was injected into the stabilizer solution maintained at $5^{\circ} \mathrm{C}$ under stirred conditions. Comminution was carried out on mechanical stirrer for a specific period at optimum speed. Continuous sonication for a specific period was applied via the probe sonicator. The temperature was maintained at $5^{\circ} \mathrm{C}$ to get less particle size. ${ }^{[16]}$ The solution was sonicated by probe sonicator for 15 minutes. The obtained nanosuspension was centrifuged using ultracentrifuge at 14000 RPM for 5 minutes at $5^{\circ} \mathrm{C}$. The supernatant solution was replaced by a stabilizer solution with appropriate concentration. To store it for a longer period it was lyophilized using lyophilizer. [17]

\section{Plackett-Burman design}

Plackett-Burman design was applied to screen out the significant parameter out of various processing parameters. The processing parameters screened were Drug Conc. in $\mathrm{mg} / \mathrm{ml}$, Solvent/Antisolvent Ratio, 
Stirring Speed, Conc. of Poloxamer 188 and Conc. of Tween 80 indicated in table 1 . The effect of each parameter evaluated using particle size, entrapment efficiency, and in vitro drug release. A total of 12 experimental trials were carried out with 5 independent factors and 3 dependant factors generated by Design-Expert ${ }^{\circledR}$. The levels of each variable were selected based on preliminary studies.

Table 1: Experimental factors and values

\begin{tabular}{cccc}
\hline \multirow{2}{*}{ Independent factors } & Coded & \multicolumn{2}{c}{ LEVELS } \\
\cline { 3 - 4 } & value & $\mathbf{- 1}$ & $\mathbf{+ 1}$ \\
\hline Drug Conc. & $\mathbf{X}_{\mathbf{1}}$ & 20 & 40 \\
Solvent/Antisolvent Ratio & $\mathbf{X}_{\mathbf{2}}$ & $1: 10$ & $1: 30$ \\
Stirring Speed & $\mathbf{X}_{\mathbf{3}}$ & 800 & 1200 \\
Conc. of Poloxamer 188 & $\mathbf{X}_{4}$ & 0.1 & 0.3 \\
Conc. of Tween 80 & $\mathbf{X}_{\mathbf{5}}$ & 0.05 & 0.15 \\
\hline
\end{tabular}

\section{Characterization of Cilnidipine Nanoparticles}

\section{Particle size}

Mean particle size and polydispersity index (PI) of the prepared nanosuspension and raw drug were checked by the Zetatrac instrument (microtrac, Mumbai). Whenever required suitably diluted Nanoparticles were added to the instrument test unit and estimation was done with the assistance of microtrac 10.6.1 programming.

\section{Entrapment efficiency}

For the determination of entrapment efficiency, prepared nanosuspension formulation was centrifuged at $10000 \mathrm{rpm}$ for $10 \mathrm{~min}$. The supernatant solution was analyzed by a UV-visible spectrophotometer at $240 \mathrm{~nm}$ using $1.2 \mathrm{pH}$ simulated gastric fluid as a blank. Entrapment Efficiency was calculated by the following equation. [18]

$\%$ Entrapment Efficiency $=\underline{\text { Total Drug Content in Nanoparticles }} \times 100$ The total amount of drug

\section{In-vitro Drug release}

In vitro release of drugs was performed utilizing the USP dissolution Apparatus II setup (Electrolab Dissolution Tester TDT-06P, USP). Simulated gastric fluid $(1.2 \mathrm{pH})$ was taken as a dissolution medium. The temperature was maintained at $37 \pm 0.5^{\circ} \mathrm{C}$, and the paddle speed used was $100 \mathrm{rpm}$. Cilnidipine Nanoparticles containing the equivalent of $10 \mathrm{mg}$ drug was added into the $250 \mathrm{ml}$ dissolution medium. Samples containing each of $5 \mathrm{ml}$ were withdrawn at a different time interval $(2,5,10,15,30,45,60,90$ and 120 $\mathrm{min}$ ) and passed through a $0.45 \mu \mathrm{m}$ syringe filter. The determination of the samples was done using a UV spectrophotometer at $240 \mathrm{~nm}$. [19]

\section{Solid-state characterization}

Fourier Transmission Infra-Red Spectroscopy (FTIR) FTIR analysis of pure drug CLN and CLN Nanoparticles were performed using FTIR CARY 630 (Agilent Technologies) to check the interaction between drug and excipients. The samples were added into the sample port for recording the IR spectra. The spectrum was recorded in the region $400-4000 \mathrm{~cm}^{-1}$.

Differential scanning calorimetry (DSC)
DSC study was performed to analyze the drug and excipients compatibility study of pure drug, and final formulation. DSC analysis was carried out using aluminum sample pans in Differential Scanning Calorimetry (DSC-60, Shimadzu Japan) at a heating rate of $10^{\circ} \mathrm{C}$ per minute in the range 30 to $300^{\circ} \mathrm{C}$ using differential scanning calorimetry (DSC).

\section{Scanning electron microscopy (SEM)}

The surface morphology of CLN Nanoparticles was studied with a field emission scanning electron microscopy. Before observation, the samples was fixed onto metal stubs using double-sided sticky tape previously secured onto aluminum stubs and then coated with gold under a vacuum and photographs were taken at various magnifications.

\section{RESULTS AND DISCUSSION}

\section{Preparation of CLN Nanoparticles}

Nanoparticles of CLN were prepared by the nanoprecipitation ultrasonication method. The drug was dissolved in Dimethyl sulphoxide (DMSO) solvent that was selected based on the highest solubility. The organic solution of CLN was added into an aqueous solution containing stabilizers Poloxamer 188 and Tween 80 . The resultant solution was stirred by mechanical stirrer at various speeds. The spontaneous precipitates were observed due to the instant desolvation of the drug. By using the stabilizer in the concentration range of 0.1 to $0.3 \%, 200$ to $500 \mathrm{~nm}$ particle size of Nanoparticles was formed. The stirring speed was kept in the range of 800 to $1200 \mathrm{rpm}$. The larger particle was formed when stirring speed was kept greater than 1200 and less than 800. If the antisolvent amount increases yield of Nanoparticles was decreased and the removal of the solvent becomes difficult so the ratio of antisolvent was selected from 1: 10 to 1: 30. Plackett Burman design was applied for the screening of factors in which levels of parameters selected based on preliminary trials.

Particle size determines the performance of drugs like drug solubility and dissolution behavior in media. Entrapment efficiency determines the performance of drugs like how much amount of drug entrapped in particles and dissolution at 10 minutes determines how much amount of drug released at 10 minutes.

Plackett Burman design

In the design total, 12 batches have been prepared. The Particle size, entrapment efficiency, and drug releases at 10 minutes of all 12 batches were in the range of 236$431 \mathrm{~nm}$ (table 2), $90.94 \%$ to $95.87 \%$ and $90 \%$ to $98 \%$. Here 11th batch was considered optimized with $236 \mathrm{~nm}$ particle size (figure 1), 95.57 entrapment efficiency, and $98.36 \%$ drug release at 10 minutes. The statistical analysis results (table 3 ) showed that $\mathrm{R}^{2}$ value greater than 0.9 . The $p$-value is less than 0.05 proves the significance of design. The F-Ratio was observed greater than theoretical $\mathrm{F}$ value 0.008 . The correlation of $R^{2}, \quad p$-value and $F$-Ratio had shown that linear relationship between predicted and observed value. ${ }^{[20]}$ 
The equation (III to V) according to coded value for particle size, entrapment efficiency (\%EE) and drug releases at 10 minutes $\left(\mathrm{D}_{10}\right)$ are the following (table 5).

Particle size $=352.91+33.08 \mathrm{X}_{1}-30.25 \mathrm{X}_{2}-19.91 \mathrm{X}_{3}-24.41 \mathrm{X}_{4}-19.58 \mathrm{X}_{5}$ (III)

$\% \mathrm{EE}=91.83-1.1 \mathrm{X}_{1}+1.21 \mathrm{X}_{2}+0.69 \mathrm{X}_{3}+0.95 \mathrm{X}_{4}+0.593 \mathrm{X}_{5}$

$\mathrm{D}_{10}=95.01-0.9 \mathrm{X}_{1}+1.192 \mathrm{X}_{2}+0.75 \mathrm{X}_{3}+0.97 \mathrm{X}_{4}+0.73 \mathrm{X}_{5}-$

Table 2: Plackett Burman design with responses

\begin{tabular}{ccccccccc}
\multicolumn{7}{c}{ Table 2: Plackett Burman design with responses } \\
$\mathbf{R}$ & & & & & & \\
$\mathbf{U}$ & $\mathbf{X}_{\mathbf{1}}$ & $\mathbf{X}_{\mathbf{2}}$ & $\mathbf{X}_{\mathbf{3}}$ & $\mathbf{X}_{\mathbf{4}}$ & $\mathbf{X}_{\mathbf{5}}$ & $\mathbf{R}^{1}$ & $\mathbf{R}^{2}$ & $\mathbf{R}^{3}$ \\
$\mathbf{N}$ & & & & & & & & \\
\hline 1 & -1 & -1 & -1 & 1 & -1 & $362 \pm 8.88$ & $92.46 \pm 0.077$ & $95.45 \pm 0.088$ \\
2 & -1 & -1 & 1 & -1 & 1 & $351 \pm 3.785$ & $92.78 \pm 0.035$ & $95.26 \pm 0.036$ \\
3 & 1 & -1 & 1 & 1 & 1 & $359 \pm 4.04$ & $92.04 \pm 0.03$ & $95.01 \pm 0.036$ \\
4 & -1 & 1 & 1 & -1 & 1 & $268 \pm 4.03$ & $94.09 \pm 0.037$ & $97.53 \pm 0.041$ \\
5 & -1 & -1 & -1 & -1 & -1 & $441 \pm 3.60$ & $87.76 \pm 0.2$ & $90.48 \pm 0.032$ \\
6 & 1 & -1 & -1 & -1 & 1 & $397 \pm 3.21$ & $88.81 \pm 0.050$ & $93.15 \pm 0.025$ \\
7 & 1 & 1 & -1 & -1 & -1 & $412 \pm 2.08$ & $90.94 \pm 0.025$ & $92.94 \pm 0.02$ \\
8 & 1 & -1 & 1 & 1 & -1 & $389 \pm 3.05$ & $89.83 \pm 0.04$ & $93.56 \pm 0.04$ \\
9 & -1 & 1 & -1 & 1 & 1 & $261 \pm 3.511$ & $94.96 \pm 0.032$ & $98.42 \pm 0.05$ \\
10 & 1 & 1 & 1 & -1 & -1 & $395 \pm 2.08$ & $90.86 \pm 0.026$ & $94.85 \pm 0.026$ \\
11 & -1 & 1 & 1 & 1 & -1 & $236 \pm 3.21$ & $95.57 \pm 0.037$ & $98.36 \pm 0.025$ \\
12 & 1 & 1 & -1 & 1 & 1 & $364 \pm 2.58$ & $91.86 \pm 0.025$ & $95.12 \pm 0.08$ \\
\hline
\end{tabular}

Table 3: ANOVA of particle size, entrapment efficiency, and dissolution rate at $\mathbf{1 0}$ minute

\begin{tabular}{cccc}
\hline Response & F-Ratio & $\boldsymbol{R}^{2}$ value & P-value \\
\hline Particle Size & 9.358706 & 0.941461 & 0.00843 \\
\% EE & 7.893815 & 0.931688 & 0.012901 \\
D10 & 7.52558 & 0.928694 & 0.014503 \\
\hline
\end{tabular}

Table 4: Statistical analysis of particle size, entrapment efficiency, and dissolution rate at 10 minute

\begin{tabular}{cccc}
\hline \multirow{2}{*}{ Response/ Factors } & \multicolumn{3}{c}{$\boldsymbol{p}$-value } \\
\cline { 2 - 4 } & Particle size & \% EE & D10 \\
\hline $\begin{array}{c}\text { Drug Concentraion } \\
\text { Solvent/ Antisolvent } \\
\text { Ratio }\end{array}$ & 0.008084 & 0.016569 & 0.036594 \\
$\begin{array}{c}\text { Speed } \\
\text { Concentration of } \\
\text { Poloxamer 188 }\end{array}$ & 0.011983 & 0.011105 & 0.012419 \\
$\begin{array}{c}\text { Concentration of } \\
\text { Tween 80 }\end{array}$ & 0.057735 & 0.083029 & 0.068157 \\
\hline
\end{tabular}

Table 5: Coefficient of variable for of particle size, entrapment efficiency, and dissolution rate at 10 minute

\begin{tabular}{ccccccc}
\hline Response & Intercept & $\mathbf{X}_{\mathbf{1}}$ & $\mathbf{X}_{\mathbf{2}}$ & $\mathbf{X}_{\mathbf{3}}$ & $\mathbf{X}_{\mathbf{4}}$ & $\mathbf{X}_{\mathbf{5}}$ \\
\hline $\begin{array}{c}\text { Particle } \\
\text { Size }\end{array}$ & 352.91 & 33.08 & -30.25 & -19.91 & -24.41 & -19.58 \\
$\%$ EE & 91.83 & -1.10 & 1.21 & 0.69 & 0.95 & 0.593 \\
D10 & 95.01 & -0.90 & 1.192 & 0.75 & 0.97 & 0.73 \\
\hline
\end{tabular}

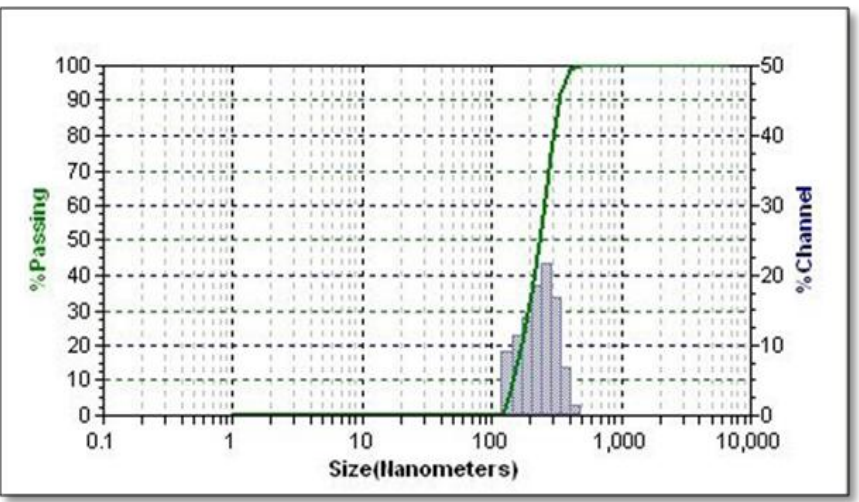

Fig. 1: Particle size distribution of Nanoparticles of Cilnidipine using zetasizer

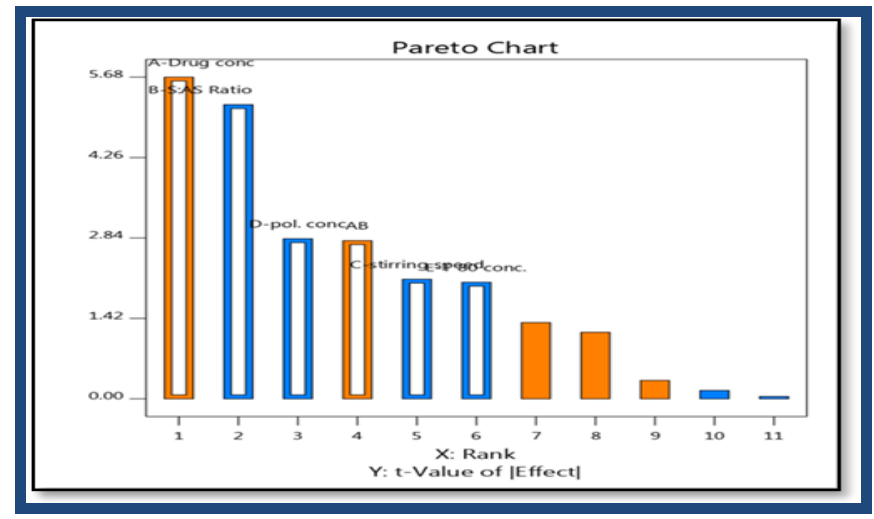

Fig. 2: Pareto chart of the effect of Independent factors on the particle size.

\section{Effect of processing variables on particle size}

The concentration of drug, solvent: antisolvent ratio and concentration of poloxamer 188 had shown $a p$ value less than 0.05 (table 4) which shows that they were the significant parameters for the method. Pareto chart (Figure 2) showed that these three variables $t$ value is also greater than 2.5 which show a significant effect on the particle size of Nanoparticles of Cilnidipine. The stirring speed and tween concentration have not significant effect on particle size. The amount of drugs has an inverse relationship with the span of the precipitated particles. The extent of the span of drug particles diminishes with an enhancement of the number of drugs. This reason is the reliance on the nucleation rate on the number of drugs in their liquid medium from which the drug is precipitate out. [21] A solvent to antisolvent proportion is a method variable, which had a negative impact on the size of the Nanoparticles. As this proportion enhances the size of Nanoparticles diminishes radically. At the point when the solution of the drug is added to the antisolvent, a fast decrease for focus happens with an expansion in the measure of antisolvent prompting quick precipitation of the drug into Nano-sized particles. At the point when the blending velocity goes higher up, the high exceptional speed delivers a lot of heat energy, which improves the temperature prompting increment in the size of Nanoparticles. [22-26] The stabilizer concentration had a negative effect on particle size as the amount of stabilizer increase than the size of particles was decreased. The scanning electron microscopy of pure drug CLN (figure 6) and Nanoparticles of CLN (figure 7) shows that there was a reduction in the size of particles of the drug.

In the Pareto plot the blue color bar shows negative effect on particle size. The orange color bar shows positive effect on particle size. White color space between bar shows that significant effect. The stirring speed and tween 80 concentration shows less significant effect on particle size. [27]

Effect of processing variables on \% Entrapment Efficiency

The concentration of drug, solvent: antisolvent ratio and concentration of poloxamer 188 had shown a $p$ - 
value less than 0.05 (table 4) which shows that they were the significant parameters for the entrapment efficiency. Pareto chart (figure 4) showed that these three variables $t$ value is also greater than 2.5 , which shows a significant effect on entrapment efficiency of Nanoparticles of Cilnidipine. The stirring speed and tween concentration have not significant effect on particle size. The drug concentration had a negative effect on entrapment efficiency as the amount of drug increases entrapment efficiency decreases. Solvent: antisolvent ratio, the concentration of Poloxamer 188, concentration of Tween 80 and stirring speed had shown a positive effect on entrapment efficiency.

The orange color was observed for solvent antisolvent ratio, stirring speed and concentration of stabilizers Poloxamer 188 and Tween 80, which indicates positive effect on entrapment efficiency. As amount of antisolvent increases, the more amount of drug can be entrapped. Drug concentration shows negative effect by the blue color bar.

Effect of processing variables on the dissolution rate at 10 minutes

The concentration of drug, solvent: antisolvent ratio and concentration of poloxamer 188 had shown a pvalue less than 0.05 (table 4 ) which shows that they were the significant parameters for the entrapment efficiency. Pareto chart (figure 4) showed that these three variables $t$ value is also greater than 2.5 , which shows a significant effect on particle size and entrapment efficiency of Nanoparticles of Cilnidipine. Drug concentration had shown a negative effect on drug release the all other variables solvent: antisolvent ratio, the concentration of poloxamer 188, concentration of tween 80 and stirring speed had shown a positive effect on drug release as per table 5 .

The orange color was observed for solvent antisolvent ratio, stirring speed and concentration of stabilizers Poloxamer 188 and Tween 80, which indicates positive effect on the dissolution rate at 10 minute. Drug concentration shows negative effect by the blue color bar. The higher stirring speed lowers the particle size and increases the drug release rate. The stabilizer amount up to certain concentration range lowers the particle size and increases the drug release rate. The higher drug concentration particle size increases and the drug release rate decreases.

Solid-state characterization

Fourier Transmission Infra-Red Spectroscopy (FTIR) Analysis

FT-IR spectra of CLN, Poloxamer 188, physical mixture and Nanoparticles of CLN are given in Figure 5. The study of IR spectra of CLN demonstrated the presence of $\mathrm{N}-\mathrm{H}$ stretching and of $\mathrm{C}=\mathrm{O}$ stretching is clearly shown by bands at $3292 \mathrm{~cm}^{-1}$ and $1697 \mathrm{~cm}^{-1}$.Strong and prominent peak at $1524 \mathrm{~cm}^{-1}$ and $1348 \mathrm{~cm}^{-1}$ confirms the existence of $\mathrm{NO}_{2}$ in the structure. Presence of secondary amine is confirmed by weak band at $3289 \mathrm{~cm}^{-1}$. Aromatic structure in the drug is confirmed by the characteristic absorption bands at $3086 \mathrm{~cm}^{-1}$ and at
1523-1491 $\mathrm{cm}^{-1}$ due to $\mathrm{C}-\mathrm{H}$ stretch and C-C stretching respectively. The spectrum illustrates $\mathrm{CH} 3$ aliphatic stretch at $2944 \mathrm{~cm}^{-1}$ along with extensive $\mathrm{NO}_{2}$ vibrations at 1577 and $1348 \mathrm{~cm}^{-1}$. The IR spectrum had showed that not any major interaction between CLN drug and its Nanoparticles.

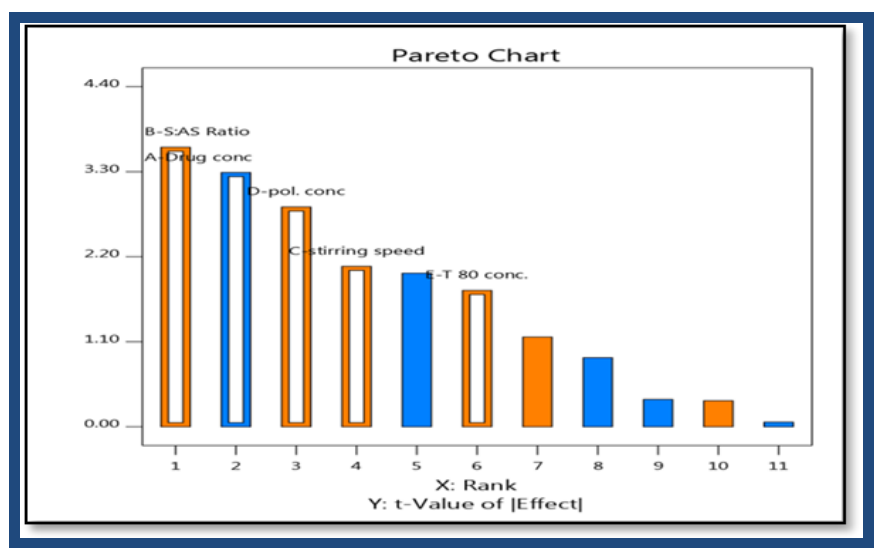

Fig. 3: Pareto chart of the effect of independent factors on the entrapment efficiency

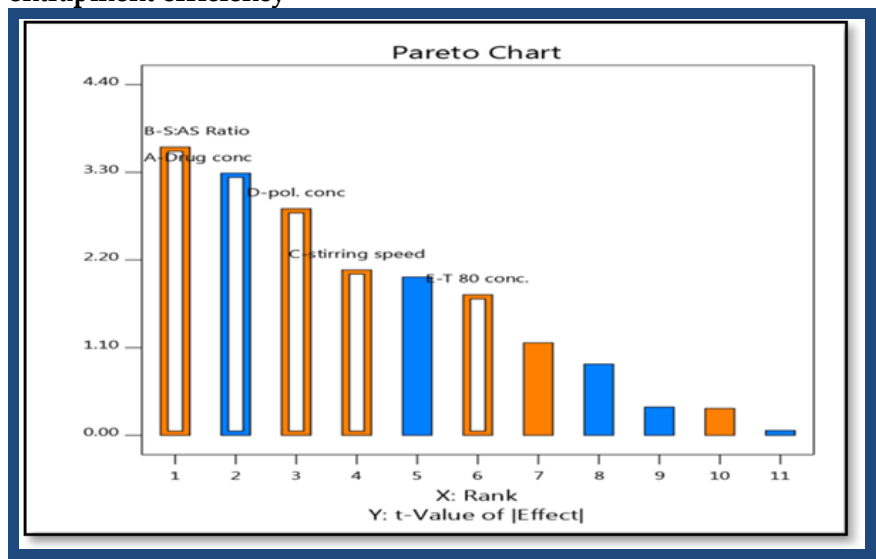

Fig. 4: Pareto chart of the effect of independent factors on the dissolution rate at 10 minute

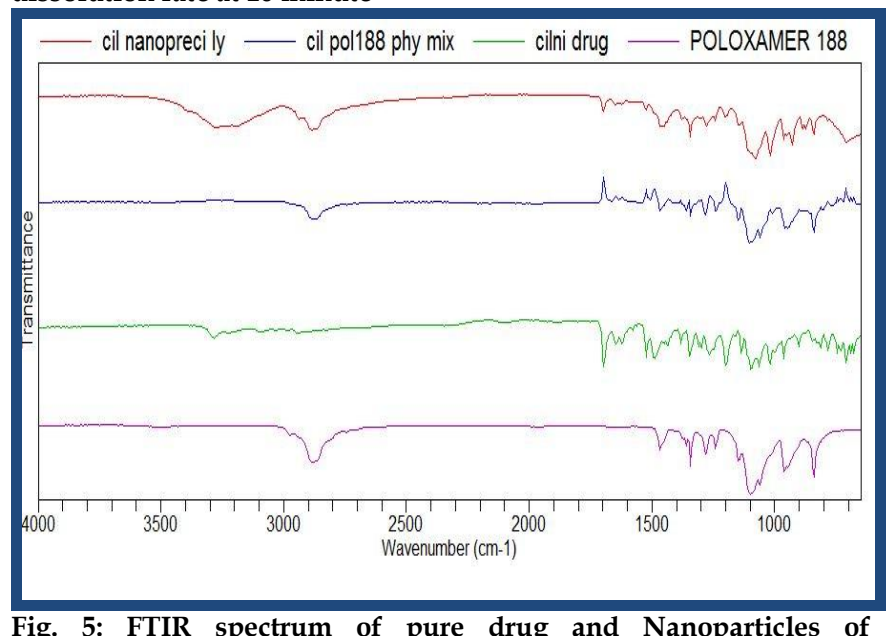

Fig. 5: FTIR spectrum of pure drug and Nanoparticles of Cilnidipine by NP-US method

\section{Differential Scanning Calorimetry (DSC) study}

The graph of the DSC pure drug CLN and CLN Nanoparticles (Figure 8 and 9) exhibited sharp endothermic melting peaks at $111.99^{\circ} \mathrm{C}$ and $112.14^{\circ} \mathrm{C}$ respectively. The graph showed no major interaction between CLN drug and CLN Nanoparticles. 


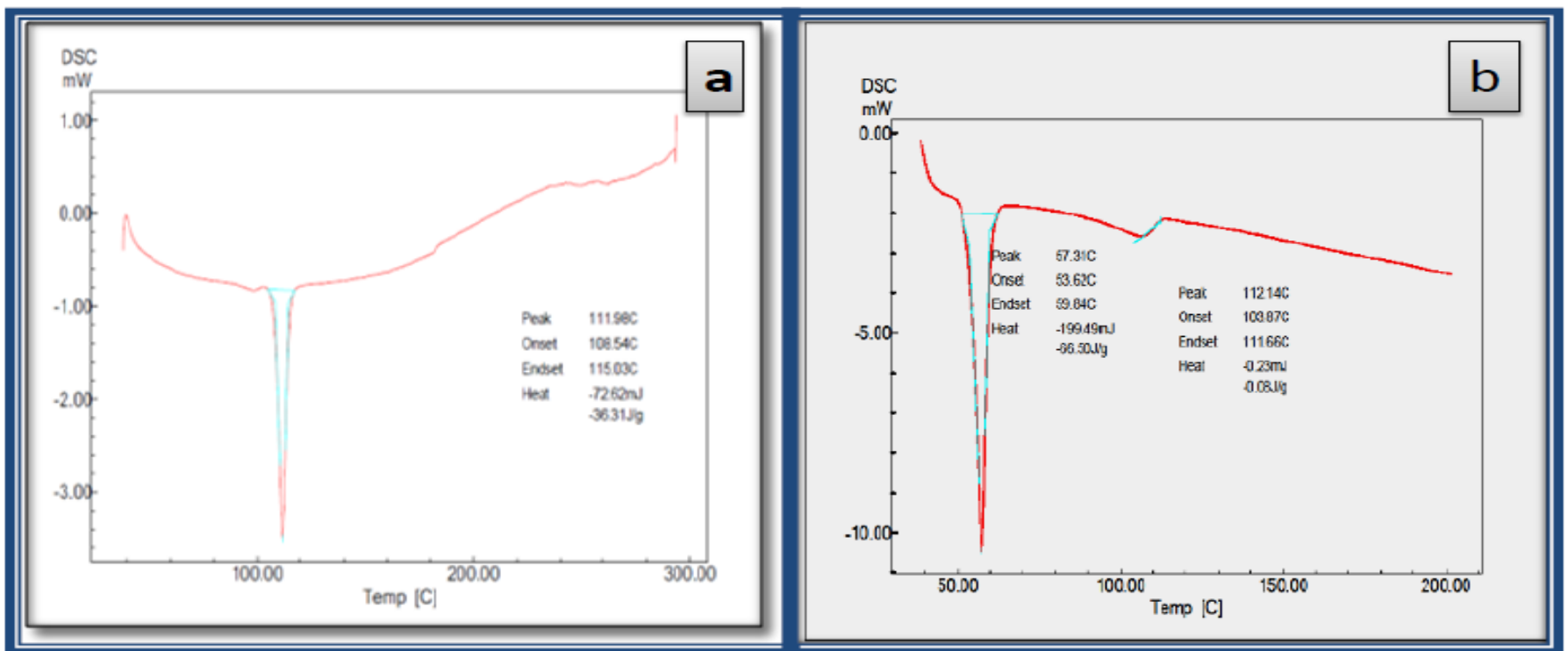

Fig. 6: DSC of pure drug CLN (a) and CLN Nanoparticles (b).

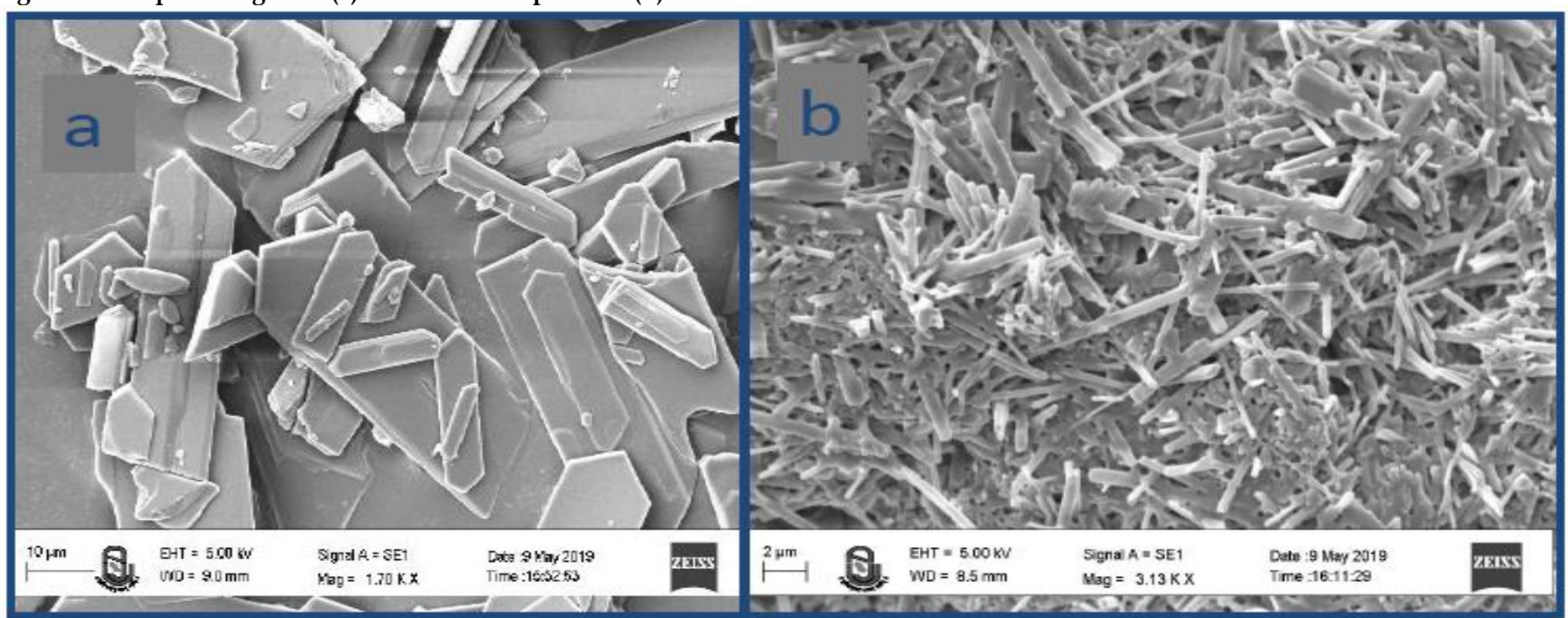

Fig. 7: scanning electron microscopic image of CLN(a) and Nanoparticles of CLN

\section{Scanning Electron Microscopy (SEM)}

Scanning electron microscopy was performed to identify the morphology of the pure drug sample and optimized CLN Nanoparticles. Scanning electron microscopy image of CLN Nanoparticles (figure 7) had shown that size of particles decreased as compare to pure drug CLN after formulating the Nanoparticles (figure 6).

In the current research, the effect of some process variables on the particle size, entrapment efficiency, and in vitro drug release of the Nanoparticles of Cilnidipine was studied by Plackett- Burman design. The lower Particle size, higher drug release, and higher entrapment of drugs can be obtained by enhancing the concentration of stabilizers, enhancing the stirring speed, enhancing the solvent: antisolvent ratio and decreasing the amount of drug.

\section{ACKNOWLEDGMENT}

I am thankful to Lakshfine chem. Pvt. Ltd and BASF for providing the gift sample of drug Cilnidipine and Poloxamer 188.

\section{REFERENCES}

1. Kitahara Y, Saito F, Akao M, Fujita H, Takahashi A, Taguchi H, Hino T, Otsuka Y, Kushiro T Kanmatsuse K. Effect of the morning and bedtime dosing with Cilnidipine on blood pressure, heart rate, and sympathetic nervous activity in essential hypertensive patients. J. Cardiovasc Pharmacol. 2004; 43: 68-73.

2. Richa M, Gautam S, Prasad K, Sahu A, Patel A, Mishra M. Solubility enhancement by solid dispersion and effervescence assisted fusion technique using Cilnidipine as a model drug, J. Chem. Pharm. Res. 2018; 10(3):51-60.

3. Tandel H, Raval K, Nayani A, Upadhay M. Preparation and evaluation of Cilnidipine microemulsion. J Pharm Bioallied Sci . 2012; 4 Suppl 1:114-5.

4. Patravale VB, Kulkarni RM. Nanosuspensions: a promising drug delivery strategy. Journal of pharmacy and pharmacology. 2004; 56(7):827-40.

5. Lipinski C. Poor aqueous solubility- an industry-wide problem in drug discovery. Am. Pharm. 2002; Rev.5: 82.

6. Patel M, Shah A, Patel NM, Patel MR, Patel KR. Nanosuspension: a novel approach for drug delivery system. Jpsbr. 2011; 7; 1(1):1-0.

7. Fessi H, Puissieux F, Devissaguet JP, Thies C. Process for the preparation of dispersible colloidal systems of a substance in the form of nanoparticles. Us Patent Us5118528, 1988. 2 June.

8. Fessi H, Devissaguet Jp, Puisieux F, Thies C. Process for the preparation of dispersible colloidal systems of a substance in the form of nanoparticles. Us Patent 593 522. 1992. 
9. Quintanar D, All'emann E, Fessi H, Doelker E. Preparation techniques and mechanisms of formation of biodegradable nanoparticles from preformed polymers. Drug Dev Ind Pharm 1998; 24:1113-1128.

10. Barichello Jm, Morishita M, Takayama K, Nagai T. Encapsulation of hydrophilic and lipophilic drugs in PLGA nanoparticles by the nanoprecipitation method. Drug Dev Ind Pharm 1999; 25:471-476

11. Rahman Z, Zidan A, Habib M, Khan. Understanding the quality of protein-loaded PLGA nanoparticles variability by Plackett-Burman design. Int. J. Pharm., 2010;389: 186-194.

12. Anastácio A, Carvalho I. Phenolics extraction from sweet potato peels: key factors screening through a placket-burman design. Ind. Crop. Prod. 2013; 43: 99-105.

13. Awotwe-Otoo D., Zidan AS, Rahman Z, Habib MJ Evaluation of anticancer drug-loaded nanoparticle characteristics by nondestructive methodologies. AAPS Pharm SciTech. 2012; 13: 611-622.

14. Singh N, Rai V. Improved antimicrobial compound production by a new isolate streptomyces hygroscopicus MTCC 4003 using Plackett-Burman design and response surface methodology. Bio information. 2012; 8: 1021-1025.

15. Ahmad M, Panda BP. Screening Of Nutrient Parameters For Red Pigment Production By Monascus Purpureus MTCC 369 Under Solid State Fermentation By Using Plackett-Burman Experimental Design. Chiang. Mai. J. Sci. 2009;36: 104-109.

16. Dengning $X$ Peng $Q$, Hongze $P$, Hongyu $P$, Shaoping $S$, Yongmei Y, Fude C. Preparation of stable nitrendipine nanosuspension using the precipitation-ultrasonication method for enhancement of dissolution and oral bioavailability. European Journal of Pharmaceutical Sciences. 2010; 40:325-334.
17. Bhanu S, Malay D. Nanoprecipitation with sonication for enhancement of oral bioavailability of furosemide, Acta Poloniae Pharmaceutica N Drug Research, 2014; 71:129-137.

18. Rohit M, Showkat R, Saima A. Polymeric nanoparticles for improved bioavailability of Cilnidipine, International Journal of Pharmacy and Pharmaceutical Sciences. 2017; 9:129-139.

19. Suparna S, Jasmine G. Development and characterization of solid self-emulsifying drug delivery system of Cilnidipine, Chem. Pharm. Bull. 2015; 63:408-417.

20. Sunny RS, Rajesh HP, Jayant RC, Navin RS. Application of Plackett-Burman screening design for preparing glibenclamide nanoparticles for dissolution enhancement, Pow. Techn. 2013; 235: 405-411.

21. Bhanu S, Malay D. Nanosuspension for enhancement of oral bioavailability of felodipine, Appl Nanosci. 2014; 9:189-97.

22. Kakran M, Sahoo N, Tan I. Preparation of nanoparticles of poorly water-soluble antioxidant curcumin by antisolvent precipitation methods. J Nanopart Res. 2012; 14:757.

23. Zhang H, Wang J, Zhang Z. Micronization of atorvastatin calcium by antisolvent precipitation process. Int J of Pharma. 2009; 374:106-113.

24. Zhang Z, Shen Z, Wang J. Nanonization of megestrol acetate by liquid precipitation. Ind Eng Chem Res. 2009; 48:84938499.

25. Wang Z, Chen J, Le Y. Preparation of ultrafine beclomethasone dipropionate drug powder by antisolvent precipitation. Ind Eng Chem Res. 2007; 46:4839-4845.

26. Kardos A, Toth J, Gyenis J. Preparation of protein loaded chitosan microparticles by combined precipitation and spherical agglomeration. Pow Tech. 2013; 244:16-25.

27. Moorthi C, Kathiresan K. Application of Plackett-Burman factorial design in the development of curcumin loaded Eudragit E 100 Nanoparticles. Nano Biomed. Eng. 2013; $5(1): 28-33$

HOW TO CITE THIS ARTICLE: Bagada A, Vadalia KR, Raval MK, Gadhia D. To Study the Significance of Processing Variables Using Quality by Design for Optimization of Nanoparticulate System of Cilnidipine. Int. J. Pharm. Sci. Drug Res. 2019; 11(6): 318-324. DOI: 10.25004/IJPSDR.2019.110606 